\title{
Rúbricas para evaluar la competencia oral en un segundo idioma: Un estudio de caso
}

Rubrics to Evaluate the Oral Competence in the Second Language: a Case Study M.Sc. Milena Barquero D’Avanzo' Mag. Elvia Ureña Salazar ${ }^{2}$

\section{Resumen}

Esta investigación analizó los instrumentos utilizados para evaluar la competencia oral de los estudiantes del Bachillerato en la Enseñanza del Inglés de la Sede del Atlántico de la Universidad de Costa Rica, por parte de sus docentes. El objetivo era proponer una herramienta más efectiva, que estuviera acorde con las actividades empleadas para fomentar esta competencia: la rúbrica. Se considera que esta herramienta propicia una mayor objetividad, pues beneficia tanto a la persona docente como al discente, porque con su uso se disminuye la posibilidad de errores evaluativos los cuales podrían estarse cometiendo por parte del profesorado. A lo largo del trabajo se explicará qué es una rúbrica así como sus características y sus ventajas, tales como la seguridad que sienten las y los estudiantes al conocer con antelación lo que se espera de ellos durante la evaluación, de igual forma, la facilidad que ofrece a las y los docentes en el momento de retroalimentar al estudiantado con respecto a su desempeño. Finalmente, se expone el proceso para la construcción de una rúbrica y se presenta una propuesta para que los docentes puedan utilizarla como guía para construir sus propios instrumentos.

\section{Palabras Clave}

Evaluación, idioma, comunicación oral, competencia oral

\footnotetext{
${ }^{1}$ Profesora de la Sede del Atlántico, Universidad de Costa Rica, mbarquero7@gmail.com

${ }^{2}$ Profesora de la Sede del Atlántico, Universidad de Costa Rica, elviaurenasalazar@gmail.com
} 


\section{Abstract}

This research analyzed the instruments used by the English Teaching instructors at the University of Costa Rica Atlantic Campus to evaluate the students' oral competence. The main objective was to propose a more effective tool accordingly to the activities used to promote this competence: the rubric. This instrument fosters more objectivity benefiting not only the instructor but also the learner, since its use decreases the possibility of making evaluative mistakes that teacher might be committing. Through the development of this research, the authors will explain what a rubric is, as well as its characteristics and such advantages as the confidence students have when they know in advance what is expected from them during the evaluation activity. They will also emphasize the ease offered to the teachers to provide feedback to the learners in regards to their performance. Finally, an explanation of the construction process of a rubric is presented, as well as an example to be used as a guide for teachers to construct its own tools.

\section{Key Words}

Evaluation, language, oral communication, oral competence

\section{La Enseñanza del Inglés en la Universidad de Costa Rica}

Esta disciplina académica tiene una gran tradición en la sociedad costarricense. En 1825, con el Decreto Ejecutivo del Reglamente Interno de la Casa de Enseñanza de Santo Tomás, se inicia el estudio de las lenguas en forma institucional. En 1954, la Universidad de Costa Rica ofreció por primera vez cursos de capacitación para los y las docentes de inglés. Ya para 1957, se profesionalizó la Enseñanza del Inglés con la apertura de la carrera de Inglés en la Facultad de Educación de la Universidad de Costa Rica (Córdoba, Coto y Ramírez, 2005, en Ureña y Ureña, en prensa).

Actualmente, la institución ofrece dos planes de estudios. En la Sede Rodrigo Facio, campus central universitario, se ofrece el Bachillerato en la Enseñanza del Inglés. Es una carrera que está bajo la responsabilidad de la Escuela de Formación Docente, de la Facultad de Educación y de la Escuela de Lenguas Modernas, de la Facultad de Letras. El otro plan homónimo se ofrece en la Sede de Occidente desde el año 1992. Esta unidad académica es la responsable del programa de estudios que en la actualidad se imparte en las sedes regionales. En esta sede se ofrece a sus estudiantes el Bachillerato y la Licenciatura en la Enseñanza del Inglés, mientras que en la Sede del Atlántico y la del Caribe, se imparte solamente el nivel de bachillerato. 


\section{La Enseñanza del Inglés en la Sede del Atlántico}

El programa "Bachillerato en la Enseñanza del Inglés", se oferta en el Recinto de Turrialba de la Sede del Atlántico en forma ininterrumpida desde 1995. En 1998, se autorizó impartirlo en el Recinto de Paraíso. En este recinto se dejó de ofertar solo una vez, para ofrecer el Bachillerato en Inglés, desconcentrado de la Sede Rodrigo Facio. En el caso del Recinto de Guápiles, la carrera se imparte desde el año 2000, con la única excepción del 2002, año en el que se suspendió la oferta por razones presupuestarias.

En total, para el año 2014, se han graduado 442 profesionales en esta área en los tres Recintos de la Sede del Atlántico. La Tabla 1 detalla el número de personas graduadas por recinto.

Tabla 1

Número de personas graduadas en Enseñanza del Inglés, Sede del Atlántico

$\begin{array}{lc}\text { Recinto } & \begin{array}{c}\text { Personas } \\ \text { graduada }\end{array} \\ \text { Turrialba } & 181 \\ \text { Paraíso } & 154 \\ \text { Guápiles } & 107\end{array}$

Fuente: Oficina de Registro e Información, Sede del Atlántico (2014)

\section{La Comunicación Oral en el Plan de Estudios}

Según el Documento Oficial de la Carrera Bachillerato y Licenciatura en la Enseñanza del Inglés, publicado por el Departamento de Filosofía, Artes y Letras de la Sede de Occidente, el plan de estudios de estas carreras plantea la necesidad de que los estudiantes adquieran conocimientos de las estructuras, pronunciación, vocabulario y demás habilidades lingüísticas y sociolingüísticas fundamentales de la segunda lengua. Se pretende además que al finalizar este programa de estudios, las y los estudiantes demuestren un dominio oral de la lengua inglesa que le permita expresarse y comunicarse adecuadamente para lograr desenvolverse en diferentes campos de trabajo. De igual manera, a lo largo del plan de estudios se procura desarrollar, en los y las estudiantes, las cuatro habilidades de un idioma, a saber: comunicación escrita, comunicación oral, lectura y escucha. 
Para el presente trabajo, las investigadoras se enfocaron en aquellos cursos cuyo primer objetivo es el desarrollo de la competencia oral. El plan de estudios cuenta con seis cursos de comunicación oral, distribuidos en los tres primeros años de la carrera; cada uno se acompaña de su respectivo laboratorio. Es importante tener en consideración que, aunque en estos cursos la habilidad que mayor se promueve es la de producción oral, también se procura desarrollar las otras habilidades, tales como la comunicación escrita, la escritura, y la lectura en un segundo idioma. Con el propósito de analizar la promoción en cada uno de los cursos de comunicación oral en los tres recintos, se solicitó a la Oficina de Registro e Información de la Sede del Atlántico, el número de estudiantes matriculados, y de estudiantes que aprobaron cada curso, durante los años 2010, 2011, 2012, 2013 y 2014. Para efectos de esta investigación, solamente se tomaron en cuenta los que finalizaron el semestre. La Tabla 2 detalla el número de alumnos reprobados en los cursos de comunicación oral durante esos tres años, en comparación con aquellos que los matricularon. Las cifras corresponden a los tres Recintos de la Sede del Atlántico.

Tabla 2

Estudiantes que matricularon y aprobaron los cursos de comunicación oral en los años 2010, 2011, 2012, 2013, y 2014

\section{Nombre del Curso}

Primer año de carrera

IO 5400: Comunicación Oral I

IO 5410: Comunicación Oral II

\section{Segundo año de carrera}

IO 5420: Comunicación Oral III

IO 5430: Comunicación Oral IV

Tercer año de carrera

IO 5440: Comunicación Oral V

IO 5450: Comunicación Oral VI

\section{Estudiantes \\ Matriculados}

\section{Estudiantes que reprobaron}

380

275

246

226

205

200

145

48

56

33

22

15
$38.15 \%$

\section{Porcentaje de \\ reprobación}

$17.45 \%$

$22.76 \%$

$14.60 \%$

$10.73 \%$

$0.07 \%$

Fuente: Elaboración propia a partir de la información aportada por la Oficina de Registro e Información, Sede del Atlántico (2014)

La información detallada en la Tabla 2 permite observar que los cursos en los cuales hay mayor nivel de reprobación son los correspondientes al primer ciclo lectivo de cada año, mientras que en los cursos correspondientes al segundo, esta es menor. Una explicación para lo anterior es que, 
según la percepción de los mismos estudiantes y del cuerpo docente, en cada nivel se cambia la actividad de comunicación que se fomentará en el aula (trabajo en parejas, presentaciones orales individuales y debates, entre otras). Destaca además, el alto nivel de reprobación en el primer ciclo. Se considera que lo anterior se debe a que la mayoría del estudiantado proviene de instituciones educativas en las cuales no se fomenta de una manera efectiva la comunicación oral en inglés.

Por otra parte, generalmente, algunas actividades de evaluación oral se realizan en clase, frente a la persona docente y al grupo de compañeros y compañeras. Las pruebas parciales y finales se realizan en parejas o en grupo pero con la presencia de al menos dos docentes, conocidos como tribunales, en apego al Artículo 20, del Reglamento de Régimen Académico Estudiantil. Durante estas pruebas, las y los compañeros de clase no se encuentran en el aula.

La anterior situación incide en la reprobación tan alta en los primeros cursos de carrera, dado que muchos de las y los educandos se enfrentan por primera vez a este tipo de actividades. Por su parte, en los cursos avanzados, los estudiantes están más familiarizados con esta dinámica de clase y de evaluación.

\section{Actividades de clase que fomentan la competencia oral}

Se analizó cada uno de los programas de los cursos de comunicación oral con el propósito de identificar las actividades utilizadas por el docente, a fin de promover la competencia oral. Y se logró determinar que en todos los cursos se utilizan actividades de escucha, de repetición, así como la imitación de sonidos y entonación. Aunque estas estrategias son muy valederas para sentar las bases de una correcta pronunciación, se debe tener cuidado con respecto al tiempo de clase que se destina para estas actividades, ya que por sí mismas no promueven la competencia oral. La misma documentación menciona que se utilizan actividades de producción controlada y libre, así como situaciones comunicativas, sin detallar si estas actividades se realizarán en pareja o en grupo.

Es oportuno destacar que para los cursos de primer año se indica el uso de actividades de producción controlada y libre, mientras, en los cursos avanzados se menciona la realización de debates orales sobre temas de importancia para las y los estudiantes. Sin embargo, para las investigadoras si se fuera más específico en el tipo de actividades por utilizar en cada curso, las cuales deben incrementar el nivel de complejidad conforme se avanza en el plan de estudios, se promovería una mayor homogeneidad entre las competencias adquiridas por las y los estudiantes de toda la Sede del Atlántico. 
En consecuencia, una mayor especificidad con respecto a las actividades comunicativas que se utilizan en cada curso, permitiría al docente a cargo y a las y los estudiantes profundizar en cada una. Se promovería la práctica intensiva en clase y se permitiría la elaboración de un instrumento de evaluación claro para las partes.

De igual manera, actividades comunicativas bien diseñadas y bien ejecutadas ayudarían al estudiantado a adquirir la confianza necesaria para participar en clase de una manera activa y segura, dado que se promueve el uso del lenguaje auténtico en situaciones muy similares a las del mundo real, al que se deben enfrentarse las y los futuros docentes. Por ejemplo, las actividades del primer curso pueden ser de trabajo individual y en parejas sobre temas del ambiente cercano al estudiantado. En el segundo curso se puede promover el trabajo en parejas pero sobre temas en los que las y los estudiantes deban hacer investigación y dar su opinión al respecto. El grado de complejidad de la tarea se incrementaría conforme se avanza en la carrera. De esta manera, se evitaría que solamente se trabaje un tipo de actividad comunicativa a lo largo de la mayoría del plan de estudios, tal y como está sucediendo con el trabajo en parejas.

\section{Metodología utilizada}

En la investigación que antecede a este documento, las autoras hicieron uso del enfoque de investigación acción, que es un medio que promueve la reflexión para así mejorar el quehacer académico. Siguiendo con este marco de referencia, se continúa con la puesta en marcha de la propuesta elaborada anteriormente.

Esto permite la generación de nuevos conocimientos para el investigador y para los grupos involucrados; moviliza y refuerza las organizaciones de base y, finalmente, hace un mejor empleo de los recursos disponibles con base en el análisis crítico de las necesidades y las opciones de cambio (Carvajal, 2008, p.31).

Se procedió a recolectar y analizar los instrumentos utilizados por el personal docente para evaluar la competencia oral de los y las estudiantes. Esto permitió visualizar el estado actual del componente de evaluación en los cursos orales y proponer estrategias que conlleven a un proceso evaluativo más objetivo. Se espera que estas alternativas sean adoptadas de forma consensuada por el cuerpo docente de toda la Sede del Atlántico. 


\section{Instrumentos utilizados para evaluar la competencia oral}

Se solicitó a los y las docentes a cargo de los cursos de comunicación oral, los instrumentos que utilizaban durante la evaluación de cada una de las actividades. El análisis de estos documentos arrojó que si bien cada uno tenía formato similar al de una rúbrica, carecían de descripciones detalladas sobre los niveles de evaluación a partir de criterios establecidos con anterioridad. Se considera que al carecer de criterios de evaluación y de descriptores claros, estas herramientas presentan dificultad para su aplicación por parte de los docentes y para su interpretación por los estudiantes. Destaca además que ningún instrumento permitía detallar el objetivo de la evaluación. Lo anterior se debe a que eran utilizados para evaluar todas las actividades del curso, sin tomar en cuenta que estas podían ser diferentes.

\section{Aspectos evaluados}

Todos los cursos de comunicación oral evaluaban los siguientes aspectos: fluidez, estructura, habilidades de comunicación, contenido, vocabulario y pronunciación. El porcentaje otorgado a cada uno de estos componentes variaba en cada instrumento de acuerdo con el profesor que lo estuviera utilizando, no necesariamente de acuerdo con la actividad de evaluación. Tampoco se tomaba en cuenta en el plan de estudios el nivel de las y los estudiantes. Por ejemplo, al componente "contenido" un instrumento le daba el valor de 15 puntos, mientras que otro se evaluaba en conjunto con el vocabulario, con un valor de 35 puntos. La misma situación se dió con la "estructura"; un instrumento la valoró con 25 puntos y el otro con 30 . Con respecto a los criterios de evaluación, algunos instrumentos utilizaban la siguiente escala: 1 = pobre; 2 = necesita mejorar; 3 = aceptable; 4 = bueno; y $5=$ excelente, mientras que otros tenían los siguientes indicadores: excelente, muy bueno, bueno, justo, y pobre, pero ninguno describía lo que se entendía por cada uno de ellos. En otras ocasiones, la escala era de 5 a 10, donde 5 era el puntaje mínimo otorgado y 10 el máximo. El o la estudiante que obtenía el puntaje máximo en cada criterio, obtenía un 100; mientras que el que obtenía la nota mínima en todos, alcanzaba un 50. También se encontraron rúbricas que utilizaban la escala de 0 a 10.

Se detectó una resistencia por parte de las y los docentes para utilizar los instrumentos que habían seleccionado para evaluar a sus estudiantes. A pesar de que ese instrumento tenía puntajes específicos para cada criterio evaluado, las y los profesores se inclinaban por otorgar una nota que fueran intermedios entre los valores incluidos en la tabla. Además, ellos informaron que sentían necesidad de estar haciendo la conversión del citado rubro a 100 puntos, para estar seguros del valor que se le estaba dando a cada componente. Posteriormente, hacían la conversión de esa nota al puntaje que en el instrumento se otorgaba a cada criterio. Finalmente, sumaban todos los 
puntajes otorgados para saber la nota obtenida en la prueba. Lo anterior evidencia la dificultad enfrentada por las y los docentes a la hora de utilizar estas escalas. Se considera que si estas fueran más claras el profesorado no presentaría tanta resistencia como la descrita anteriormente. Otros instrumentos subdividían en categorías cada criterio de evaluación, a saber: fluidez, estructura, habilidades de comunicación, contenido y vocabulario, pronunciación. Por ejemplo, las habilidades comunicativas, las cuales, cada una recibía un puntaje, se dividían en lenguaje corporal y fluidez. En otro caso, para la evaluación del trabajo en grupo específicamente, las dos grandes áreas: contenido y desarrollo, se dividían a su vez en diferentes categorías tales como: introducción, cuerpo de la conferencia, organización y uso del lenguaje. También se detectó que algunos de los instrumentos analizados incluían categorías o descriptores que no estaban necesariamente presentes en la actividad de evaluación, pero que tenían un puntaje en la escala. Según los docentes entrevistados, al no estar presente en la actividad de evaluación, y al estar incluidos en la herramienta, estos criterios recibieron el puntaje máximo.

La información anterior evidencia la falta de un instrumento estándar en la Sede del Atlántico que pueda ser utilizado por su cuerpo docente, para evaluar la competencia oral en inglés del alumnado. Este instrumento sería sujeto de cambios menores en el valor de cada criterio, de acuerdo al nivel del estudiante y al curso en que se encuentra, pero sería igual, o al menos similar, en cada uno de los cursos de comunicación oral de los tres Recintos de esta Sede Universitaria. De esta manera, se procuraría al máximo que los instrumentos utilizados estén adecuados al tipo de tarea que se está siendo evaluada. Se hace necesario, entonces, otorgarles las herramientas para evaluaciones pertinentes y oportunas para cada curso y actividad que se realicen.

El propósito de este artículo es presentar para consideración de la comunidad docente una propuesta de modelo de evaluación de la competencia oral, que se fundamente en el uso de las rúbricas. La propuesta del instrumento evaluador incluye el objetivo de evaluación, el cual también debe estar acorde con la actividad misma de evaluación, así como con el nivel de competencia del estudiantado. Además, debe establecer los criterios de evaluación de una forma clara, de manera tal que sean entendidos por la persona docente como por las y los estudiantes. Estas características básicas permitirían cumplir con lo solicitado por el Artículo 20 del Reglamento de Régimen Académico Estudiantil, a saber: "Los miembros del tribunal evaluador deberán indicarle al estudiante los objetivos y criterios por evaluar, al inicio de la realización de la prueba". Se recomienda que el instrumento sea discutido con el estudiantado por lo menos ocho días antes de la prueba oral. 
Las rúbricas son instrumentos válidos para evaluar muchas actividades educativas; sin embargo, se considera que son el instrumento más adecuado para evaluar la competencia oral de una persona. Como se ha venido insistiendo, al elaborar esta herramienta, se debe tomar en cuenta el objetivo planteado para cada actividad de evaluación, así como a la tarea específica que deberán realizar los estudiantes durante la clase. Se recomienda además, que las actividades comunicativas que se utilizan como medio para promover la competencia oral en las y los alumnos en cada una de las lecciones, sean iguales a las que se utilizan para evaluar esta capacidad. Si se cumpliera con estas características, las herramientas serían de fácil manejo por parte de la persona docente y de las y los estudiantes. De esta manera, se cumpliría con lo señalado por el Artículo 20 del Reglamento anteriormente mencionado, con respecto a la necesidad de brindar al estudiantado una constancia sobre el lugar, hora y fecha de la realización del examen, así como la calificación obtenida.

\section{Marco Teórico}

\section{La rúbrica}

Existen varios instrumentos de evaluación que pueden adaptarse para valorar la competencia oral de las y los aprendices. Uno de ellos es la rúbrica, instrumento que se define como la guía precisa para la valoración de los aprendizajes y productos realizados por las y los estudiantes. Este instrumento desglosa los niveles de desempeño de las y los aprendices en un aspecto determinado, con criterios específicos sobre rendimiento; además, indica el logro de los objetivos curriculares y las expectativas de las y los docentes. Permite, además, las y los estudiantes identifiquen con claridad la relevancia de los contenidos y los objetivos de los trabajos académicos establecidos (Gatica y Uribarren, 2013). Citando a Diaz-Barriga (2006), Gatica y Uribarren (2013) señalan que las rúbricas facilitan la valoración en áreas consideradas como subjetivas, complejas o imprecisas, por medio de criterios que cualifican progresivamente el logro de aprendizajes, conocimientos y/o competencias valoradas desde un nivel incipiente hasta uno experto. La evaluación de la competencia oral es difícil porque es un proceso complejo en el que intervienen situaciones que pueden ser consideradas subjetivas o imprecisas, tales como el nerviosismo de las y los estudiantes y el cansancio de las personas miembros del tribunal, entre otras. Por otro lado, para Rimari (2008) una rúbrica representa una descripción en palabras de las características esenciales que debe tener un logro o desempeño, considerado como un conjunto integrado de aprendizajes. La persona docente, entonces, debe procurar el logro de una descripción clara de lo que se espera de las y los estudiantes con respecto a cada uno de los criterios incluidos, de manera tal que se minimice al máximo toda posibilidad de incongruencia y falta de claridad. 
Para efectos de este estudio, se conceptualizó como rúbrica a la combinación de criterios y estándares que se utilizan para evaluar el nivel de desempeño de las y los estudiantes en la competencia oral a través de tareas $\mathrm{y} / \mathrm{o}$ actividades diseñadas para tal efecto. Aunque existen las rúbricas holísticas y las analíticas, para Martínez (2008), la segunda es la más adecuada para evaluar las actividades que se desarrollan en los cursos estudiados, dado que este tipo de instrumento permite identificar las necesidades, fortalezas y deficiencias del estudiante. Este tipo de instrumento facilitaría el proceso de realimentación que se da a la hora de entregar los resultados obtenidos en el examen.

Aunque para el autor, las rúbricas se pueden utilizar cuando se necesite dar un valor a las siguientes actividades: informes orales o escritos, portafolios, observaciones y ejercicios de ejecución, entre otros; en el caso que nos ocupa, se propone que se utilice este instrumento para evaluar las estrategias de comunicación utilizadas por las y los estudiantes en los cursos de Comunicación Oral del programa "Bachillerato en la Enseñanza del Inglés", que se ofrece en los Recintos de Turrialba, Paraíso y Guápiles, de la Sede del Atlántico de la Universidad de Costa Rica.

\section{¿Por qué utilizar rúbricas?}

De acuerdo con Martínez (2008), dentro de las principales razones por las cuales las rúbricas deben ser utilizadas se encuentran que en ellas se formula y se fundamenta las características que debe tener un buen producto final, la posibilidad de las y los estudiantes para conocer de antemano la forma en que serán evaluados, la facilidad que brindan a la hora de dar al estudiante realimentación sobre sus fortalezas y debilidades, así como la reducción de la subjetividad a la hora de evaluar. En resumen, las rúbricas son excelentes instrumentos de evaluación, pues se adecúan perfectamente para los propósitos de valorar la competencia oral de los aprendices de un segundo idioma.

También se recomienda el uso de la rúbrica en las ocasiones en las que se está aplicando la técnica Aprendizaje Basado en Problemas (ABP) porque en esta práctica el escenario puede ser confuso para la persona aprendiz, por lo tanto la rúbrica le indicará claramente lo que debe concretar, así como el nivel de exigencia cuando reporte esa actividad, así se disminuye el estrés, el cual puede provocar fallas en las y los estudiantes (López, 2009). En el caso particular de la comunicación oral en inglés, se considera que el escenario puede ser confuso pues pueden intervenir factores que vayan a afectar positiva o negativamente el desempeño del aprendiz. Por lo tanto, un diseño apropiado de este instrumento de evaluación ayudará a aminorar la incertidumbre del alumno sobre la forma en que se le evaluará. 
Si bien López (2009) recomienda entregar al alumno la totalidad de las rúbricas que se van a utilizar en cada curso, de ser posible durante la primera semana de clase, para las investigadoras es suficiente dar a conocer y discutir las rúbricas con los estudiantes, por lo menos con una semana de antelación a la prueba. Además, dadas las características de los cursos, sujeto de este estudio, se recomienda utilizar los mismos instrumentos para evaluar tanto las actividades de clase como los tribunales. De esta forma, el grupo de usuarias y usuarios estará familiarizado con los instrumentos.

Las rúbricas pueden ser globales o analíticas. Con las globales también conocidas como comprensivas y holísticas, se valora, el desempeño del estudiante de una forma integrada . No se determinan los componentes del proceso o temas evaluados. Se permite una valoración general con descriptores correspondientes a niveles de logro sobre calidad, comprensión o dominios globales. Demandan menor tiempo para calificar, pero la retroalimentación es limitada (Goodrich, 2005, en Gatica y Uribarren, 2013). A partir de la anterior descripción, se presenta la Figura 1, una propuesta de rúbrica global que permita valorar la competencia oral de una persona ubicada en el curso Comunicación Oral I, durante una actividad en parejas.

Figura I

Propuesta de rúbrica global para evaluar la competencia oral del aprendiz principiante

Universidad de Costa Rica - Sede del Atlántico

Recinto de Guápiles / Recinto de Paraíso/ Recinto de Turrialba

Curso: 105400 Comunicación Oral I

Actividad de Clase

Evaluación Parcial Evaluación Final

Objetivo: Evaluar la capacidad del aprendiz para mantener una conversación con su compañero (a), por lo menos durante cinco minutos, sobre el tema indicado.

Modalidad: Conversación espontánea en parejas.

Fecha: Nombre del estudiante:

Puntaje Total: 5 Puntaje Obtenido: Nota: 


\begin{tabular}{|c|c|c|}
\hline Escala & Nombre & Descripción \\
\hline 5 & $\begin{array}{l}\text { Avanzado/ } \\
\text { Excelente }\end{array}$ & $\begin{array}{l}\text { Evidencia comprensión de la tarea asignada. Se expresa } \\
\text { correctamente en inglés sobre el tema indicado con facilidad y } \\
\text { espontaneidad. Utiliza efectivamente un vocabulario variado y } \\
\text { demuestra conocimiento del uso indicado de las diferentes } \\
\text { estructuras gramaticales. La participación es balanceada con } \\
\text { respecto a su pareja. }\end{array}$ \\
\hline 4 & $\begin{array}{l}\text { Intermedio/ } \\
\text { Sobresaliente }\end{array}$ & $\begin{array}{l}\text { Evidencia comprensión de la tarea asignada. Se expresa } \\
\text { adecuadamente en inglés sobre el tema indicado con cierto grado de } \\
\text { facilidad y espontaneidad. Utiliza efectivamente un vocabulario } \\
\text { variado y demuestra conocimiento del uso indicado de las diferentes } \\
\text { estructuras gramaticales. La participación es balanceada con } \\
\text { respecto a su pareja. }\end{array}$ \\
\hline 3 & $\begin{array}{l}\text { Medio / } \\
\text { Aceptable }\end{array}$ & $\begin{array}{l}\text { Evidencia comprensión parcial de la tarea asignada. Se expresa } \\
\text { correctamente en inglés sobre el tema indicado con alguna facilidad } \\
\text { y espontaneidad. Utiliza un vocabulario adecuado y variado. } \\
\text { Demuestra conocimiento del uso indicado de las diferentes } \\
\text { estructuras gramaticales. La participación no es balanceada con } \\
\text { respecto a su pareja. }\end{array}$ \\
\hline 2 & $\begin{array}{l}\text { Básico / } \\
\text { Deficiente }\end{array}$ & $\begin{array}{l}\text { Evidencia poca comprensión de la tarea asignada. Se expresa con } \\
\text { dificultad en inglés sobre el tema indicado. Utiliza vocabulario y } \\
\text { estructuras gramaticales de forma limitada. La participación se centra } \\
\text { en una persona. }\end{array}$ \\
\hline 1 & $\begin{array}{l}\text { Inicio / } \\
\text { Insuficiente }\end{array}$ & $\begin{array}{l}\text { No hay evidencia de comprensión de la tarea asignada. El uso del } \\
\text { idioma inglés es muy limitado. Hay poca participación. }\end{array}$ \\
\hline 0 & & No realizó la tarea asignada. \\
\hline
\end{tabular}

Fuente: Elaboración propia, a partir de Gatica y Uribarren (2013) y Martínez (2008)

Un factor importante de este tipo de rúbrica es que se puede graduar los diferentes niveles de desempeño, con el fin de obtener suficientes criterios o elementos que definen determinado nivel. Se incluye un nombre para que las y los estudiantes entiendan con mayor facilidad lo que significa cada nivel (Martínez, 2008). Se pretende que, a partir del ejemplo anterior, las y los docentes desarrollen más rúbricas que estén acordes con el objetivo de la actividad y que sean compartidas con el estudiantado, por lo menos con una semana de antelación. La propuesta de las investigadoras es que las y los profesores de los tres recintos trabajen en conjunto, de manera tal 
que se logre uniformar los criterios de evaluación de la competencia oral en cada uno de los cursos en estudio.

La rúbrica analítica, por su parte, se utiliza para evaluar los componentes del desempeño del estudiante, los cuales sirven como base para la calificación total. Shipman (2012), en Gatica y Uribarren (2013), señala que esta modalidad de rúbrica puede utilizarse para determinar de una forma más específica las fortalezas y debilidades del desempeño de las y los estudiantes. La retroalimentación que reciben es más completa. Este tipo de rúbrica detalla los criterios para evaluar. También cada criterio puede subdividirse de acuerdo con la profundidad requerida.

Es oportuno tener en consideración que el análisis de los instrumentos utilizados por el cuerpo docente de la Sede del Atlántico para evaluar la competencia oral, arrojó los siguientes criterios de evaluación como los más comunes: fluidez, contenido y vocabulario, gramática, pronunciación y habilidades de comunicación. También se encontró que había variación entre el valor otorgado a cada uno de estos componentes.

La Figura 2 muestra una propuesta de rúbrica analítica para evaluar a una pareja de estudiantes mientras desarrolla una conversación a partir del tema propuesto por el / la docente, en la cual se evaluará los criterios citados anteriormente. Esta rúbrica es un ejemplo en el cual los criterios o categorías de evaluación tienen un mismo peso en la nota final del examen.

Figura 2

Propuesta de rúbrica analítica para evaluar la competencia oral del aprendiz principiante

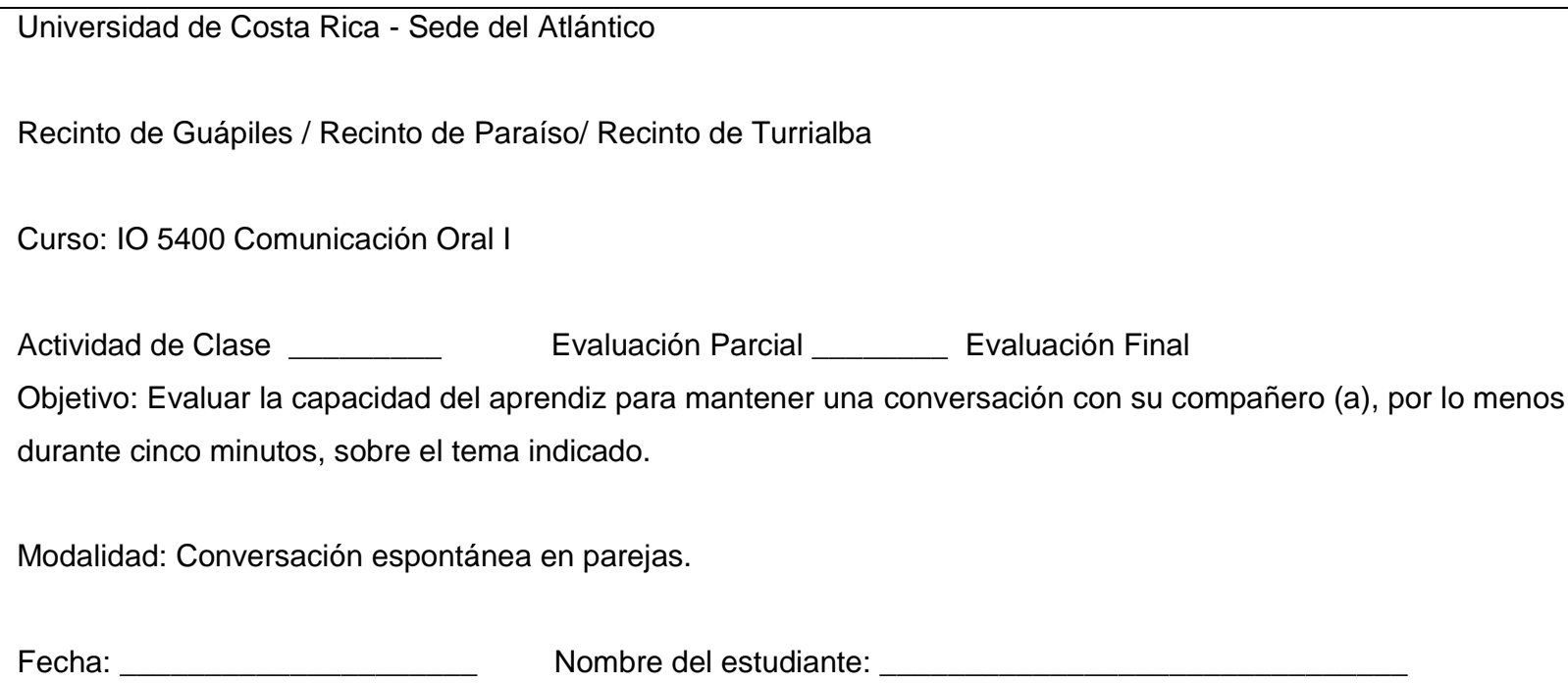


InterSedes. Vol. XVI. (34-2015) ISSN: 2215-2458

\begin{tabular}{|c|c|c|c|c|c|}
\hline Puntaje Total: 5 & Puntaje $\mathrm{O}$ & tenido: & Nota: & & \\
\hline $\begin{array}{c}\text { Criterios o } \\
\text { categorías de } \\
\text { evaluación }\end{array}$ & $\begin{array}{c}4 \\
\text { Excelente }\end{array}$ & $\begin{array}{c}3 \\
\text { Buen trabajo }\end{array}$ & $\begin{array}{c}2 \\
\text { Aceptable }\end{array}$ & $\begin{array}{c}1 \\
\text { Necesita } \\
\text { Mejorar }\end{array}$ & $\begin{array}{c}\text { Puntaje } \\
\text { Otorgado }\end{array}$ \\
\hline Fluidez & $\begin{array}{l}\text { Se expresa } \\
\text { correctamente } \\
\text { con facilidad y } \\
\text { espontaneidad. }\end{array}$ & $\begin{array}{l}\text { Se expresa } \\
\text { correctamente } \\
\text { con facilidad y } \\
\text { espontaneidad. } \\
\text { Las pausas que } \\
\text { realiza al } \\
\text { conversar no } \\
\text { distorsionan la } \\
\text { claridad del } \\
\text { mensaje. }\end{array}$ & $\begin{array}{l}\text { Se expresa } \\
\text { con dificultad. } \\
\text { El mensaje no } \\
\text { se entiende. }\end{array}$ & $\begin{array}{l}\text { No se expresa } \\
\text { correctamente. } \\
\text { No hay } \\
\text { espontaneidad. } \\
\text { Las pausas que } \\
\text { realiza al } \\
\text { conversar } \\
\text { distorsionan la } \\
\text { claridad del } \\
\text { mensaje. }\end{array}$ & \\
\hline $\begin{array}{l}\text { Contenido y } \\
\text { Vocabulario }\end{array}$ & $\begin{array}{l}\text { Habla sobre el } \\
\text { tema indicado. } \\
\text { Aporta nuevas } \\
\text { ideas y promueve } \\
\text { la participación } \\
\text { de su pareja. } \\
\text { Utiliza } \\
\text { correctamente el } \\
\text { vocabulario } \\
\text { estudiado en } \\
\text { clase. } \\
\text { Explica los } \\
\text { nuevos } \\
\text { conceptos. }\end{array}$ & $\begin{array}{l}\text { Aunque habla } \\
\text { sobre el tema, } \\
\text { indicado no aporta } \\
\text { nuevas ideas. } \\
\text { Utiliza el } \\
\text { vocabulario } \\
\text { estudiado en } \\
\text { clase la mayoría } \\
\text { de las veces en } \\
\text { forma correcta. } \\
\text { Explica los } \\
\text { nuevos } \\
\text { conceptos, pero } \\
\text { no siempre de } \\
\text { forma correcta. }\end{array}$ & $\begin{array}{l}\text { Se limita a } \\
\text { comentar lo } \\
\text { aportado por } \\
\text { su pareja. No } \\
\text { promueve la } \\
\text { participación } \\
\text { espontánea. } \\
\text { El uso que le } \\
\text { da al } \\
\text { vocabulario } \\
\text { estudiado en } \\
\text { clase indica } \\
\text { que no conoce } \\
\text { el significado } \\
\text { de algunos } \\
\text { términos. } \\
\text { Explica los } \\
\text { nuevos } \\
\text { conceptos. }\end{array}$ & $\begin{array}{l}\text { Lo aportado no } \\
\text { está relacionado } \\
\text { con el tema } \\
\text { indicado. } \\
\text { El uso que le da } \\
\text { al vocabulario } \\
\text { estudiado en } \\
\text { clase indica que } \\
\text { no conoce su } \\
\text { significado. }\end{array}$ & \\
\hline Gramática & $\begin{array}{l}\text { Utiliza las } \\
\text { estructuras } \\
\text { gramaticales }\end{array}$ & $\begin{array}{l}\text { Frecuentemente, } \\
\text { utiliza las } \\
\text { estructuras }\end{array}$ & $\begin{array}{l}\text { La mayoría de } \\
\text { las veces } \\
\text { utiliza las }\end{array}$ & $\begin{array}{l}\text { El uso que le da } \\
\text { a las estructuras } \\
\text { estudiadas en }\end{array}$ & \\
\hline
\end{tabular}


InterSedes. Vol. XVI. (34-2015) ISSN: 2215-2458

\begin{tabular}{|c|c|c|c|c|}
\hline & $\begin{array}{l}\text { estudiadas en } \\
\text { clase sin error. } \\
\text { Se aventura a } \\
\text { utilizar } \\
\text { estructuras no } \\
\text { estudiadas en } \\
\text { clase. }\end{array}$ & $\begin{array}{l}\text { gramaticales } \\
\text { estudiadas en } \\
\text { clase, con errores } \\
\text { que no afectan el } \\
\text { mensaje. }\end{array}$ & $\begin{array}{l}\text { estructuras } \\
\text { gramaticales } \\
\text { estudiadas en } \\
\text { clase, los } \\
\text { errores afectan } \\
\text { el mensaje. }\end{array}$ & $\begin{array}{l}\text { clase indica que } \\
\text { no conoce su } \\
\text { aplicación. }\end{array}$ \\
\hline Pronunciación & $\begin{array}{l}\text { Habla claro. Casi } \\
\text { no comete } \\
\text { errores de } \\
\text { pronunciación. } \\
\text { Aplica las reglas } \\
\text { de pronunciación } \\
\text { estudiadas en } \\
\text { clase. }\end{array}$ & $\begin{array}{l}\text { Habla claro. } \\
\text { Comete errores } \\
\text { de pronunciación } \\
\text { que no afectan la } \\
\text { claridad del } \\
\text { mensaje. }\end{array}$ & $\begin{array}{l}\text { La mayoría de } \\
\text { las veces habla } \\
\text { claro. Los } \\
\text { errores de } \\
\text { pronunciación } \\
\text { que comete } \\
\text { afectan muy } \\
\text { poco la } \\
\text { claridad del } \\
\text { mensaje. }\end{array}$ & $\begin{array}{l}\text { No habla claro y } \\
\text { comete errores } \\
\text { de } \\
\text { pronunciación } \\
\text { que afectan la } \\
\text { claridad del } \\
\text { mensaje. }\end{array}$ \\
\hline $\begin{array}{l}\text { Habilidades de } \\
\text { comunicación }\end{array}$ & $\begin{array}{l}\text { Elabora al menos } \\
\text { tres preguntas } \\
\text { sobre el tema } \\
\text { indicado a su } \\
\text { pareja. } \\
\text { La conversación } \\
\text { es natural al cien } \\
\text { por ciento. }\end{array}$ & $\begin{array}{l}\text { Elabora dos } \\
\text { preguntas sobre } \\
\text { el tema indicado a } \\
\text { su pareja. } \\
\text { Duda para dar su } \\
\text { respuesta, pero } \\
\text { esto no afecta la } \\
\text { naturalidad de la } \\
\text { conversación. }\end{array}$ & $\begin{array}{l}\text { Elabora una } \\
\text { pregunta sobre } \\
\text { el tema } \\
\text { indicado a su } \\
\text { pareja. } \\
\text { Las dudas en } \\
\text { su } \\
\text { participación } \\
\text { demuestran } \\
\text { que su } \\
\text { conversación } \\
\text { no es natural. }\end{array}$ & $\begin{array}{l}\text { No hace } \\
\text { preguntas a su } \\
\text { pareja. Se limita } \\
\text { a contestar. } \\
\text { Duda al } \\
\text { contestar. El } \\
\text { tiempo de } \\
\text { espera por su } \\
\text { participación } \\
\text { refleja que su } \\
\text { conversación no } \\
\text { es natural. }\end{array}$ \\
\hline
\end{tabular}

Fuente: Elaboración propia, a partir de Gatica y Uribarren (2013)

Tanto la Figura 1, como la 2 fueron elaboradas para evaluar a estudiantes con un nivel de conocimiento de principiantes. Lo anterior, debido a que serían utilizadas en el primer curso de la carrera. El uso de la rúbrica analítica o de la global dependerá del interés particular de la persona docente. 
También hay situaciones en las que la persona docente podría necesitar darle un mayor peso a algún criterio sobre otro u otros. En esos casos, la recomendación que hace Martínez (2008, 131132) es seguir el siguiente procedimiento.

a. Definir los criterios que se evaluarán. En el caso que nos ocupa, estos criterios serían: fluidez, contenido y vocabulario, gramática, pronunciación y habilidades comunicativas.

b. Establecer el peso de cada uno de esos criterios en la nota global que recibirá el estudiante. En el ejemplo utilizado, la situación que se estableció era una conversación en parejas sobre el tema indicado, que durara por lo menos cinco minutos. Al ser los estudiantes principiantes, la persona docente podría preferir darle más importancia al contenido y vocabulario, luego a la fluidez, a las habilidades de comunicación, posteriormente a la gramática y por último a la pronunciación. Así las cosas, asignaría, en una escala de 1 a 10 , al contenido y vocabulario un valor de $10 \%$, a la fluidez de $8 \%$, a las habilidades de comunicación de $6 \%$, a la gramática de $4 \%$ y a la pronunciación de $2 \%$. Estos valores son sugerencias que se presentan para ilustrar el tipo de rúbrica analítica en discusión y el modo de aplicarla quedaría a criterio del / la docente.

c. Redactar, para cada uno de los criterios definidos, los descriptores para cada nivel de logro.

d. Cuando ya se cuenta con los descriptores de cada uno de los aspectos evaluados, se procede a elaborar una tabla de puntuaciones, de manera tal que las y los estudiantes puedan recibir una nota global de su desempeño durante la actividad de evaluación.

La Figura 3 es una propuesta de rúbrica analítica en la que los criterios de evaluación no tienen igual peso en la nota global. 
Figura 3

Segunda propuesta de rúbrica analítica para evaluar la competencia oral del aprendiz principiante

Universidad de Costa Rica - Sede del Atlántico

Recinto de Guápiles / Recinto de Paraíso/ Recinto de Turrialba

Curso: IO 5400 Comunicación Oral I

Actividad de Clase

Evaluación Parcial

Evaluación Final

Objetivo: Evaluar la capacidad del aprendiz para mantener una conversación con su compañero (a), por lo menos durante cinco minutos, sobre el tema indicado.

Modalidad: Conversación espontánea en parejas.

Fecha:

Nombre del estudiante:

Puntaje Total: 5

Puntaje Obtenido:

Nota:

\begin{tabular}{|c|c|c|c|c|c|}
\hline Puntos & $\begin{array}{l}\text { Contenido y } \\
\text { Vocabulario }\end{array}$ & Fluidez & $\begin{array}{l}\text { Habilidades de } \\
\text { comunicación }\end{array}$ & Gramática & Pronunciación \\
\hline $0-2$ & $\begin{array}{l}\text { La } \\
\text { participación } \\
\text { no es } \\
\text { suficiente para } \\
\text { evaluar }\end{array}$ & $\begin{array}{l}\text { No se expresa } \\
\text { correctamente. No } \\
\text { hay } \\
\text { espontaneidad. }\end{array}$ & $\begin{array}{l}\text { No hace preguntas } \\
\text { a su pareja. Se } \\
\text { limita a contestar. } \\
\text { Duda al contestar. } \\
\text { El tiempo de } \\
\text { espera por su } \\
\text { participación } \\
\text { refleja que su } \\
\text { conversación no } \\
\text { es natural. }\end{array}$ & $\begin{array}{l}\text { El uso que le da a } \\
\text { las estructuras } \\
\text { estudiadas en } \\
\text { clase indica que } \\
\text { no conoce su } \\
\text { aplicación. }\end{array}$ & $\begin{array}{l}\text { Habla claro, cuando } \\
\text { comete errores de } \\
\text { pronunciación, la } \\
\text { claridad del } \\
\text { mensaje no se ve } \\
\text { afectada. }\end{array}$ \\
\hline $3-4$ & $\begin{array}{l}\text { Lo aportado no } \\
\text { está } \\
\text { relacionado } \\
\text { con el tema }\end{array}$ & $\begin{array}{l}\text { Hay poca o } \\
\text { limitada } \\
\text { espontaneidad. } \\
\text { Las pausas que } \\
\text { realiza al }\end{array}$ & $\begin{array}{l}\text { Se limita a } \\
\text { comentar lo } \\
\text { aportado por su } \\
\text { pareja. No } \\
\text { promueve la }\end{array}$ & $\begin{array}{l}\text { Utiliza las } \\
\text { estructuras } \\
\text { gramaticales } \\
\text { estudiadas en }\end{array}$ & (n.... \\
\hline
\end{tabular}




\begin{tabular}{|c|c|c|c|c|c|}
\hline & $\begin{array}{l}\text { indicado. } \\
\text { El uso que le } \\
\text { da al } \\
\text { vocabulario } \\
\text { estudiado en } \\
\text { clase indica } \\
\text { que no conoce } \\
\text { su significado. }\end{array}$ & $\begin{array}{l}\text { conversar } \\
\text { distorsionan la } \\
\text { claridad del } \\
\text { mensaje. }\end{array}$ & $\begin{array}{l}\text { participación } \\
\text { espontánea. }\end{array}$ & $\begin{array}{l}\text { clase. } \\
\text { Se aventura a } \\
\text { utilizar estructuras } \\
\text { no estudiadas en } \\
\text { clase. } \\
\text { Cuando hay } \\
\text { errores, no se } \\
\text { afecta el mensaje. }\end{array}$ & 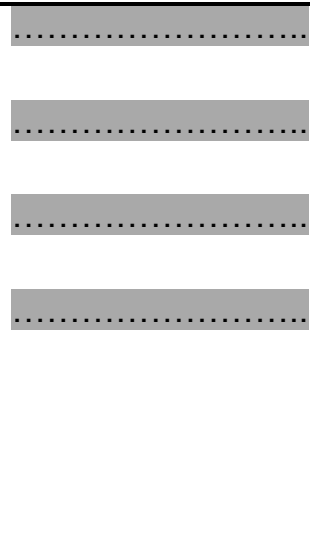 \\
\hline $5-6$ & $\begin{array}{l}\text { El uso que le } \\
\text { da al } \\
\text { vocabulario } \\
\text { estudiado en } \\
\text { clase indica } \\
\text { que no conoce } \\
\text { el significado } \\
\text { de algunos } \\
\text { términos. } \\
\text { Explica los } \\
\text { nuevos } \\
\text { conceptos. }\end{array}$ & $\begin{array}{l}\text { Se expresa } \\
\text { correctamente con } \\
\text { facilidad y } \\
\text { espontaneidad. } \\
\text { Las pausas que } \\
\text { realiza al } \\
\text { conversar no } \\
\text { distorsionan la } \\
\text { claridad del } \\
\text { mensaje. }\end{array}$ & $\begin{array}{l}\text { Elabora al menos } \\
\text { tres preguntas } \\
\text { sobre el tema } \\
\text { indicado a su } \\
\text { pareja. } \\
\text { La conversación } \\
\text { es natural. }\end{array}$ & 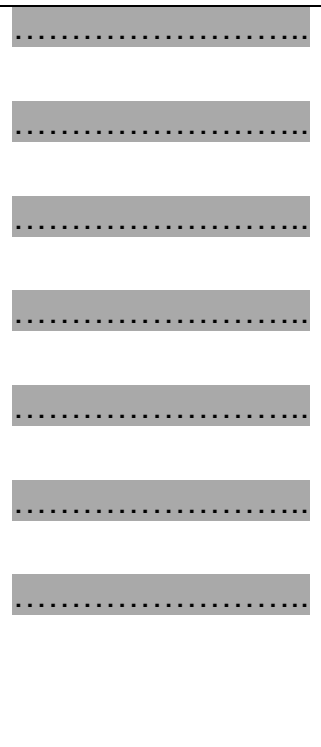 & 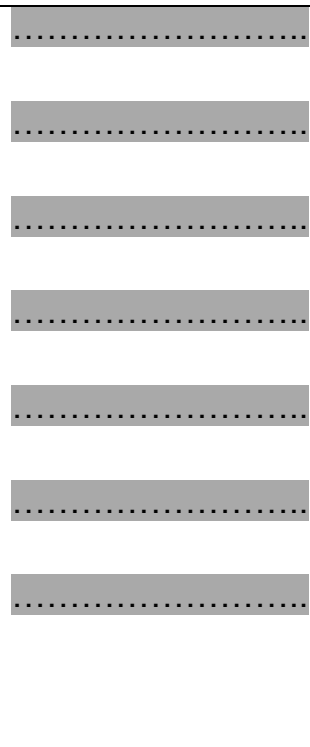 \\
\hline $7-8$ & $\begin{array}{l}\text { Aunque habla } \\
\text { sobre el tema } \\
\text { indicado, no } \\
\text { aporta nuevas } \\
\text { ideas. } \\
\text { Utiliza el } \\
\text { vocabulario } \\
\text { estudiado en } \\
\text { clase la } \\
\text { mayoría de las } \\
\text { veces en forma } \\
\text { correcta. } \\
\text { Explica los }\end{array}$ & $\begin{array}{l}\text { Se expresa } \\
\text { correctamente con } \\
\text { facilidad y } \\
\text { espontaneidad. }\end{array}$ & 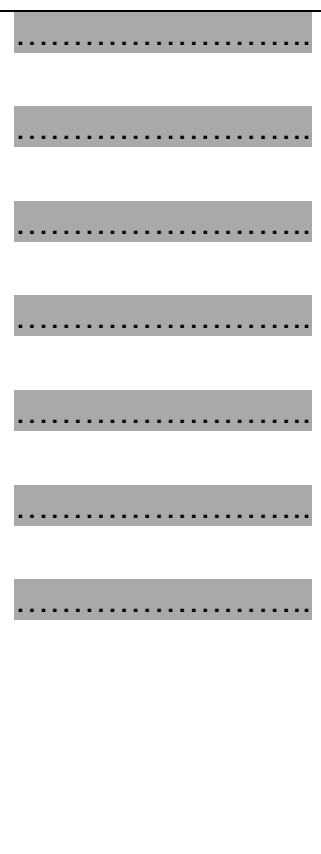 & 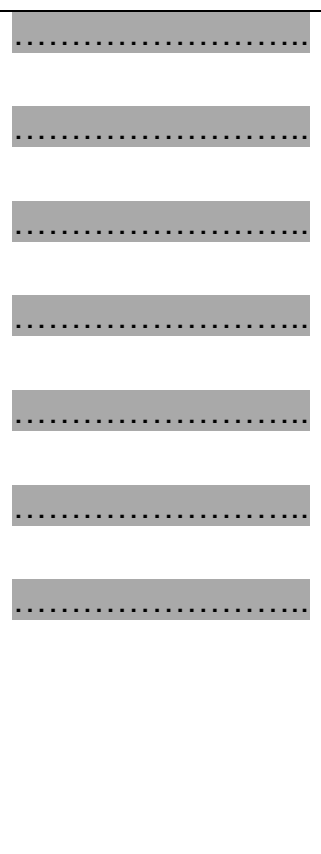 & 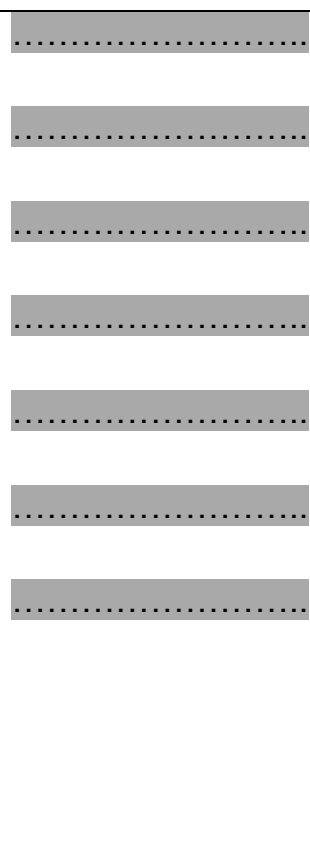 \\
\hline
\end{tabular}




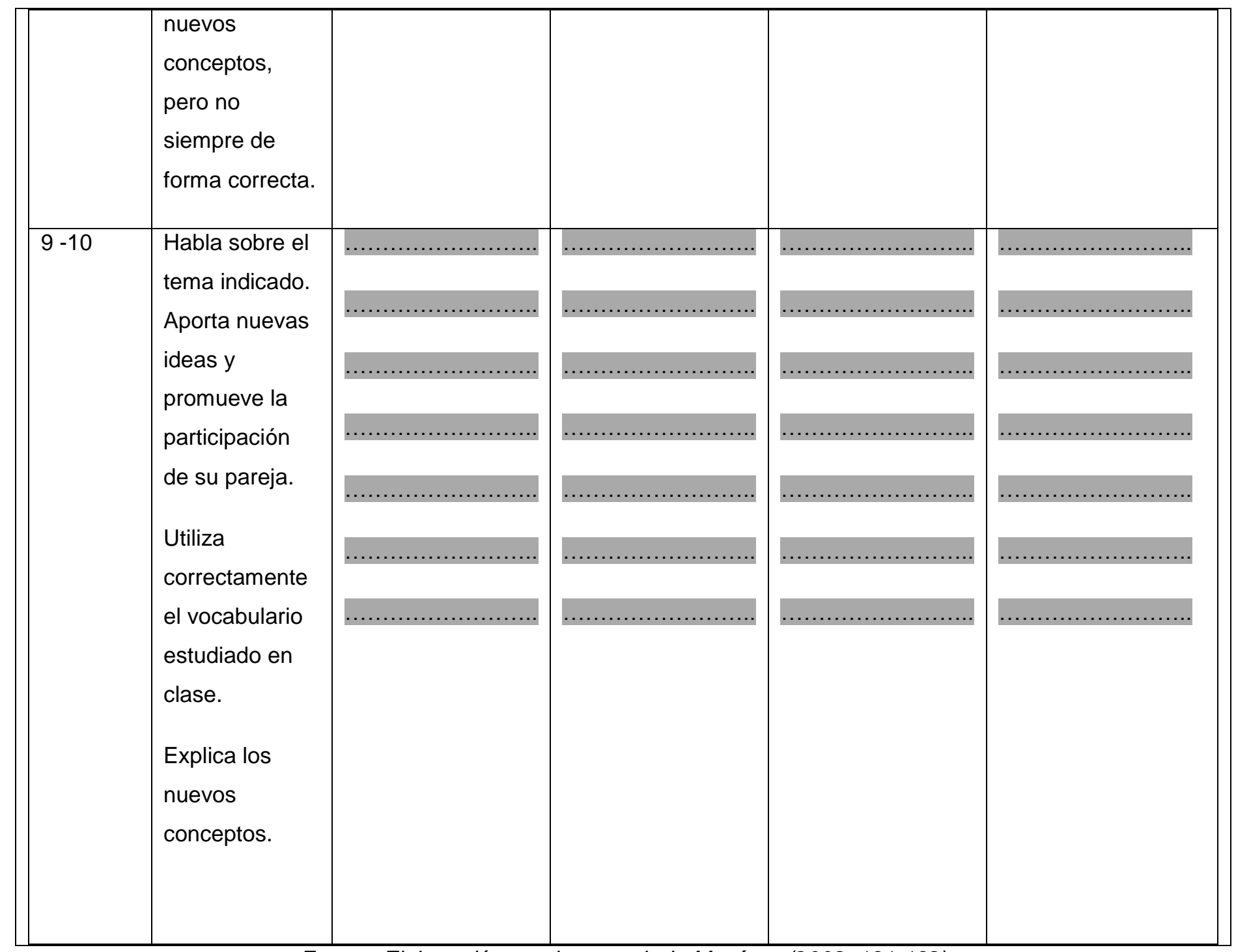

Fuente: Elaboración propia, a partir de Martínez (2008, 131-132)

e. Cuando se quiere dar una nota global a partir de la aplicación de la rúbrica anterior, lo que procede es organizar la calificación obtenida, de manera tal que se adecue a la escala numérica de 0 a 10, establecida en el Reglamento de Régimen Académico Estudiantil, de la Universidad de Costa Rica. La Tabla 4 es un modelo para obtener esta nota. 
Tabla 4

Modelo de tabla de puntuaciones y nivel correspondiente

$\begin{array}{ccc}\text { Puntos Obtenidos } & \text { Nota } & \text { Escala Numérica } \\ 24-30 & \text { Excelente } & 9.5-10 \\ 16-23 & \text { Muy Bueno } & 8.5-9 \\ 10-15 & \text { Bueno } & 7.5-8 \\ 5-9 & \text { Suficiente } & 6.5-7 \\ 0-4 & \text { Insuficiente } & 5.5-6\end{array}$

Fuente: Elaboración propia a partir de Martínez $(2008,132)$

Sin importar el tipo de rúbrica que se escoja, Gatica y Uribarren (2013) recomiendan que el instrumento incluya (a) los criterios de evaluación; es decir, los factores que determinarán la calidad del trabajo de la persona evaluada; (b) las definiciones de calidad o explicaciones detalladas de qué se espera que realice el / la estudiante; y (c) las estrategias de puntuación, las cuales deben presentarse en niveles para facilitar la comprensión del grupo de usuarios. Aunque se considera que las rúbricas son escalas ordinales que destacan una evaluación del desempeño centrada en aspectos cualitativos, es posible establecer puntuaciones numéricas. (Martínez, 2008). Al incluir puntuaciones numéricas en las rúbricas propuestas, es más fácil para las y los docentes y estudiantes adecuar las calificaciones obtenidas a la escala numérica de la Universidad de Costa Rica.

\section{Conclusiones}

La evaluación de la competencia oral es una tarea compleja, tanto para el cuerpo docente como para las y los estudiantes. Se debe evaluar no solo lo que la persona aprende a partir de los objetivos planteados, sino también la manera en que transmite el mensaje en el idioma que se está aprendiendo. Por lo cual, se considera que con el uso de rúbricas como las sugeridas en el presente artículo, se minimizan aquellos factores que atentan contra la objetividad y efectividad de la tarea evaluadora. Se recomienda que el estudiantado conozca, por lo menos, una semana antes de la actividad de evaluación, el instrumento que será utilizado. De esta manera se le otorga la posibilidad de conocer y valorar con anterioridad el qué y el cómo será evaluado y se disminuye los niveles de estrés a la hora de que el estudiantado está siendo evaluado. Además, se fortalece el estado emocional de la persona evaluada, pues elimina la incertidumbre a la que se somete por falta de instrumentos pertinentes para la evaluación. 
Al elaborar la rúbrica, la cual debe ser específica para cada tarea, el profesional en ejercicio determinará las características del producto final que se requiere, según el área que se desea evaluar. Así, se podrá conocer con claridad el nivel de exigencia de cada estrategia de evaluación. Lo anterior le permitirá a la persona evaluadora mayor objetividad para valorar las competencias y una mejor adaptación a los requerimientos específicos para cada actividad. Además, se evitará confusión en los criterios de evaluación, lo que puede afectar el desempeño del aprendiz. Finalmente, este instrumento faculta al profesional y /o al tribunal de para mejorar los procesos de evaluación, por lo cual constituye una herramienta pertinente en la enseñanza de un segundo idioma, pues provee al evaluador de niveles, indicadores y criterios de evaluación definidos. Como parte del proceso de evaluación, la misma rúbrica facilitará la realimentación con las y los estudiantes sobre sus fortalezas y debilidades en su desempeño en un segundo idioma.

\section{Bibliografía}

Carvajal, V. (2008). Módulo Didáctico: Investiguemos. Centro de Investigación y Docencia en Educación. Universidad Nacional, Heredia, Costa Rica: Programa de Publicaciones e Impresiones UNA.

Consejo Universitario, Universidad de Costa Rica (2001). Reglamento de Régimen Académico Estudiantil. Unidad de Información. Recuperado de:

http://www.cu.ucr.ac.cr/normativ/regimen_academico_estudiantil.pdf

Gatica, F. y Uribarren, T. (2013). ¿Cómo elaborar una rúbrica? Investigación en Educación Médica. Inv. Ed. Med. 2013; 2(1):61-65. Recuperado de:

http://riem.facmed.unam.mx/sites/all/archivos/V2Num01/10_PEM_GATICA.PDF

López, J.L. (2009). Uso de rúbricas generalizadas para evaluar conocimientos en la técnica didáctica Aprendizaje Basado en Problemas. Departamento de Ingeniería Mecánica, ITESM, Campus Monterrey. Recuperado de: www.mty.itesm.mx/rectoria/dda/rieee/word-files/1B-Uso-

\section{Rubricas.doc}

Martínez, J.G. (2008). Las rúbricas en la evaluación escolar: Su construcción y su uso.

Avances en Medición, 6: 129-134. Recuperado de:

http://www.humanas.unal.edu.co/psicometria/files/9713/7036/4861/Encuesta_Cuestionario_O_Prue ba.pdf 
Oficina de Registro e Información, Sede del Atlántico, Universidad de Costa Rica. (2013). Promoción de los estudiantes inscritos en los cursos orales. Bachillerato en la Enseñanza del Inglés: 2010-2011-2012. Cartago, Costa Rica.

Rimari, W. (2008). La rúbrica. Un innovador y eficaz instrumento de evaluación. Revista Pedagógica del Maestro Peruano. San Jerónimo. Marzo. Recuperado de http://es.scribd.com/doc/16918521/LA-RUBRICA-instrumento-de-evaluacion-Wilfredo-Rimari

Ureña E. y Ureña V. (en prensa). Las Competencias Genéricas en la formación de las y los estudiantes de la enseñanza del inglés en la Universidad de Costa Rica: visión de docentes y estudiantes y su relación con las demandas de los entes empleadores. Revista Umbral. 